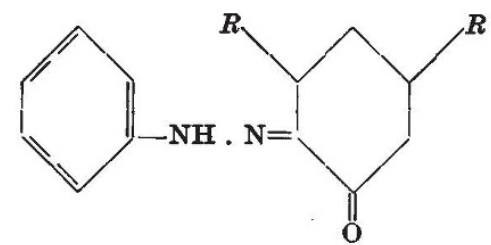

I

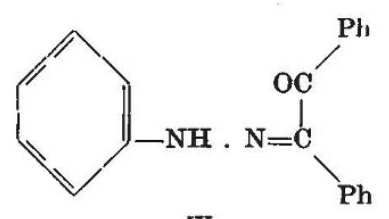

IV

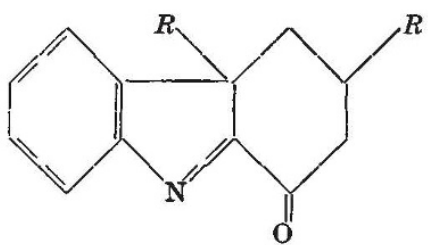

II

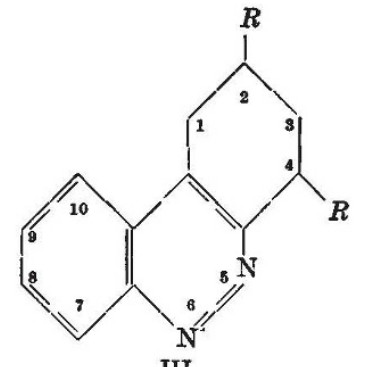

III<smiles>c1ccc(-c2nnc3ccccc3c2-c2ccccc2)cc1</smiles>

$\mathbf{V}$<smiles>NC1=C(C=Cc2ccccc2)C=C[IH]C=C1</smiles>

in yields of 75 per cent of theory. This compound has already been described by Simpson ${ }^{2}$, who obtained it from $\alpha$ (2-aminophenyl)- $\alpha$ : $\beta$-diphenylethylene (VI) by diazotization.

When applicable, the new method illustrated by the above examples is more convenient than those previously available.

I am indebted to Sir Robert Robinson for his interest in the investigation.

Dyson Perrins Laboratory,

B. P. MOORE

Oxford.

Dec. 17.

${ }^{1}$ Coffey, S., Rec. trav. chim., 42, 528 (1923).

'Simpson, J. C. E., J. Chem. Soc., 447 (1943).

\section{Influence of Solvent Movement on Sedimentation}

ENoKssoN ${ }^{1}$ has maintained that the major factor which causes the sedimentation constant of a protein to change with concentration is the counterflow of solvent, which is a necessary result of the sedimentation of the protein. He ascribed to the same cause the differences of sedimentation-rate in terms of which two of $u^{2}$ have accounted for the boundary anomalies observed in the sedimentation of mixtures. Pedersen $^{3}$ has repeated Enoksson's arguments.

$W^{2}$ were aware of this effect; but we consider that it can account only for a minor part of the change of sedimentation-rate with concentration, on the following grounds :

(1) The values of $\phi$, the specific sedimentation volume, which have to be assumed are unreasonably large, and, moreover, are inconsistent with each other and with other data. For example, the value of $\varphi$ obtained by Enoksson from our data ${ }^{2}$ on the variation of the sedimentation constant of serum albumin with concentration should be $5.4 \mathrm{ml}$./gm. (Enoksson, in his Table 1, wrongly quotes a value of $10 \mathrm{ml} . / \mathrm{gm}$.$) ;$ the value obtained for serum albumin from the boundary anomaly in mixtures with lactoglobulin is correctly calculated by Enoksson as about $11 \mathrm{ml}$./gm. ; the maximum value consistent with the sedimentation constant of serum albumin at zero concentration $\left(4.5 \times 10^{-13}\right)$, by applying Stokes's law, is $1.5 \mathrm{ml} / \mathrm{gm}$.

(2) The sedimentation constant should, on this theory, vary linearly with concentration down to zero concentration, whereas Pedersen ${ }^{4}$ has shown that, in the case of lactoglobulin, the variation becomes very small at concentrations less than $1 \mathrm{gm} . / 100 \mathrm{ml}$., and this finding has been confirmed recently ${ }^{5}$. There is no evidence that lactoglobulin dissociates under these conditions.

(3) The explanation offered does not account for the fact ${ }^{2}$ that a protein which sediments through a solution of a less rapidly sedimenting protein (that is, the faster component of a mixture) does so at the same rate as it would do in the same total concentration of itself alone : the counterflow must be less in the former case than in the latter (ef. paragraph 1).

(4) No account is taken of the proximity of the sedimenting particles to each other ; they are treated as though each were sedimenting against the counter. flow of a uniform medium of infinite extent. The effect of the proximity of neighbouring particles on their viscous movement must be an important one, though it appears that no satisfactory quantitative treatment can be given at the present time.

Enoksson further maintained that the counterflow effect can account for apparent deficiencies of the amount of a sedimenting protein, as computed from the sedimentation diagram. No deficiency is, in fact, found, provided that the solvent which is displaced is in Donnan equilibrium (which will normally be the case) and that the value used for the specific refractive increment of the protein is obtained by measurement against dialysate. Any deficiency is likely to be due to use of a wrong value for the specific refractive increment and to errors affecting the baseline of the sedimentation diagram ${ }^{6}$.

Finally, we should point out that our account ${ }^{2}$ of the boundary anomaly is independent of any particular assumption about the cause of variation of sedimentation-rate with concentration.

R. Cercil

J. P. Johnston

A. G. Ogston

Department of Biochemistry, Oxford.

Dec. 16.

1 Nature, 161, 934 (1948).

2 Johnston, J. P., and Ogston, A. G., Trans. Farad. Soc., 42, 789 (194k).

s Pedersen, K. O., "Ann. Rev. Biochem." (1948).

- Pedersen, R. O., Biochem. J., 30, 961 (1936)

5 Cecil, R., and Ogston, A. G., Biochem. J., 44, 33 (1949).

'Cecil, R., and Ogston, A. G., Biochem. J., 43, 592 (1948). 